\title{
On the Resolution Improvement of Radar Target Identification with Filtering Antenna Effects
}

\author{
Lakkhana Bannawat, ${ }^{1}$ Akkarat Boonpoonga ${ }^{D},{ }^{1}$ Santana Burintramart, ${ }^{2}$ \\ and Prayoot Akkaraekthalin ${ }^{1}$ \\ ${ }^{1}$ Electrical Engineering, Department of Electrical and Computer Engineering, Faculty of Engineering, King Mongkut's University of \\ Technology North Bangkok, Bangkok, Thailand \\ ${ }^{2}$ Defence Technology Institute (Public Organization), Ministry of Defence, Nonthaburi, Thailand
}

Correspondence should be addressed to Akkarat Boonpoonga; akkarat.b@eng.kmutnb.ac.th

Received 26 August 2017; Revised 12 December 2017; Accepted 26 December 2017; Published 28 February 2018

Academic Editor: Jaume Anguera

Copyright ( 2018 Lakkhana Bannawat et al. This is an open access article distributed under the Creative Commons Attribution License, which permits unrestricted use, distribution, and reproduction in any medium, provided the original work is properly cited.

\begin{abstract}
An investigation on the improvement of the resolution of a radar target identification system is presented in this paper. Degradation of resolution is mainly due to influence factors associated with antennas, including the strong coupling between transmitting and receiving antennas and the variation in the antenna response. A filtering technique was therefore introduced to mitigate the underlying problem. In the technique, the antenna effects were filtered out of the total response backscattered from the objects in the radar target identification system. The short-time matrix pencil method (STMPM) was then employed to extract the poles from the backscattered response in order to identify the object. Simulation and experimentation examples are illustrated to confirm the improvement of the resolution by filtering the antenna effects. The simulation and experimentation were divided into several categories, that is, different antennas and differently shaped objects, in order to validate the advantage of filtering the antenna effects. They were setup in order to demonstrate that the poles obtained from performing the STMPM without the filtering technique were mainly because of the antenna rather than the object's characteristic. The results showed that the resolution of the identification was significantly increased when performing pole extraction and filtering the antenna effects.
\end{abstract}

\section{Introduction}

Since over half a century, radar target identification systems have been intensely investigated in research areas of electromagnetic (EM) wave propagations and signal processing. There are many potential applications of the system, such as air defense, ground penetrating radar (GPR), and chipless RFID identification systems [1-5]. The challenging problem in the system is identifying unknown objects with different shapes and constituents, buried or not underground. The singularity expansion method (SEM) is one of the most employed methods to characterize the electromagnetic response of a radar target. The SEM was first introduced by observing the time-domain signal backscattered from the target [6]. By illuminating the target with the wideband EM pulse, the scattered response in the time domain is composed of two successive parts, namely, early-time and late-time responses. The first portion comes from the direct reflection of the incident EM pulse on the target. The second is due to the resonance phenomena of the target. A key to the SEM is to model the late-time portion as a sum of the complex exponentials, called the poles. Therefore, there is a need for a scheme to be employed in order to extract the poles from the late-time response.

The most popular method that has been widely employed for pole extraction is the matrix pencil method (MPM) because of its performance in dealing with noise [7]. However, the main drawback of applying MPM for identification is that it requires correct isolation between the early-time and late-time responses. In 2008, the time frequency domain was used to observe the evolution of partial resonances in the early-time responses and the commencement of the latetime response [8]. Thus, this approach can overcome the weakness of requiring the correct isolation between the 
early-time and late-time responses. Recently, the short-time matrix pencil method (STMPM) has been also proposed to overcome this weakness by sliding a proper time window of the conventional MPM's (5). It has been applied for detection in chipless RFID systems. An alternative method proposed in [9], based on a sliding-window analysis, applied the Kronecker's theorem to determine the characteristic changes in the Hankel matrix, including the matrix rank and the distribution of eigenvalues. The change is an intrinsic indicator of the stage of the target response that is currently windowed. This novel method can determine the commencement of the late-time response without a priori knowledge of the target geometry or orientation. More recently, we proposed a simple technique to estimate the commencement of the late-time response by using a cross correlation between the backscattering and transmitted waves [10]. Using the STMPM along with the proposed late-time estimation, the extracted poles will converge to a stable constant value at the position of the superimposed pattern between the transmitted waves and the late-time response. Thus, the correct extracted poles can be achieved without human skill or experience. This proposed technique was then applied to the GPR system in order to achieve the automatic detection and classification [11].

Besides to the precise pole extraction, suitable RF components are required for the radar target identification system. A popular solution is to use ultra-wideband components, including antennas and RF chain [12]. Unfortunately, the use of ultra-wideband components is a high-cost solution and designing microwave components that possess the characteristics of no variation in the ultra-wideband frequency range becomes a more serious problem. An alternative candidate has been proposed to mitigate the antenna effects by using the deconvolution technique $[13,14]$. In addition, antenna calibration was also employed to resolve the problems of antenna dispersion and multiple reflections in radar systems $[15,16]$. However, the antennas used in these literatures were designed for a particular purpose, especially radar systems and their bandwidths were ultra-wideband. For example, the horn antenna can operate in the frequency range of $800 \mathrm{MHz}$ to $10 \mathrm{GHz}$ [15]. Moreover, the effect of the use of different antenna types has not been evaluated, and nobody has seriously taken the pole resolution into account. A solution to resolve this problem due to the antenna effects is introduced here along with the investigation conducted for the pole resolution improvement.

In this paper, we present the investigation of the resolution improvement in terms of poles for a radar target identification system. A filtering technique will be introduced to mitigate the ground bounce and antenna effects due to coupling between transmitting and receiving antennas and the variation in the antenna response. The STMPM was employed to extract poles from the scattering response. A few of the antennas under consideration was the off-theshelf products which were not designed for the radar applications. The performances of the radar identification system using off-the-shelf and not off-the-shelf antennas were compared. The simulation and experimentation were setup to demonstrate if the resolution degradation was mainly

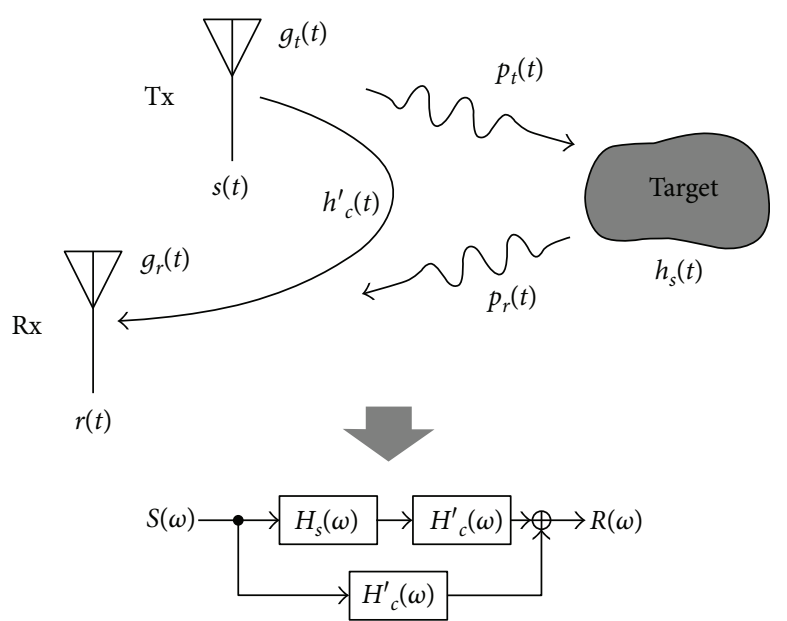

Figure 1: Basic model of a bistatic radar system.

because of the antenna effects rather than objects. The results show that the resolution of the identification is then significantly increased when performing pole extraction along with filtering the antenna effects. Following this, filtering the antenna effects for a bistatic radar system is then introduced along with derivation of its equations in Section 2, in which a target identification technique based on the STMPM is discussed. Simulations of the target identification with and without filtering the antenna effects are presented in Section 3. The experimentation results are also shown in order to confirm the superiority of the proposed approach in Section 4. Finally, conclusions are drawn in Section 5.

\section{System Model}

2.1. Filtering the Antenna Effects for the Bistatic Radar. Antennas are essential components in a radar target identification system. Typically, there is a variation in the antenna response throughout the range of operating frequency. The influence factor associated with antennas results in not only waveform distortion but also resolution degradation in target identification. In this section, a mathematical description of the proposed technique being employed to resolve the underlying problem is given. Figure 1 shows a basic model of a bistatic radar system. The receiving $(\mathrm{Rx})$ antenna is placed with the transmitting $(\mathrm{Tx})$ antenna as a bistatic radar configuration. The electromagnetic (EM) pulse $s(t)$ is fed to the transmitting antenna, whose impulse response is $g_{t}(t)$. When the output signal of the transmitting antenna strikes an object which is modeled by the impulse response of $h_{s}(t)$, the signal scattered from the object will be received by the receiving antenna whose impulse response is $g_{r}(t)$. The scattering signal $r(t)$ received by the receiving antenna contains the desired information of the object characteristic and the undesired information, such as the free space and antenna effect, as expressed as

$$
\begin{aligned}
r(t)= & s(t) * g_{t}(t) * p_{t}(t) * h_{s}(t) * p_{r}(t) * g_{r}(t) \\
& +s(t) * g_{t}(t) * h_{c}(t) * g_{r}(t),
\end{aligned}
$$


where $p_{t}(t)$ and $p_{r}(t)$ are the transfer functions of free space for the incident and reflected signals, respectively. The $h_{c}$ is the loss between the transmitting and receiving antennas, and "*" denotes and the convolution. Reconsidering Figure 1, the generated signal $s(t)$ was transmitted by using the transmitting antenna $g_{t}(t)$ and then propagated through the free space channel $p_{t}(t)$ to strike an object $h_{s}(t)$. The signal backscattered from the object propagates through the free space channel $p_{r}(t)$ and was then received by the receiving antenna $g_{r}(t)$. This behavior corresponds to the first term in (1). On the other hand, the transmitted signal corresponding to $s(t) * g_{t}(t)$ directly propagates to the receiving antenna. This direct path from the transmitting to the receiving antenna corresponds to the second term in (1).

Based on the SEM principle, the extracted complex frequency is a tool for aspect-independent target identification. Thus, the free space loss does not affect the extracted complex frequency. Their transfer functions can be omitted from the equation, which is rewritten as

$$
r(t)=s(t) *\left\{g_{t}(t) * h_{s}(t) * g_{r}(t)+g_{t}(t) * g_{r}(t)\right\} .
$$

Here, the term $g_{t}(t)^{*} g_{r}(t)$ includes the mutual coupling between the transmitting and receiving antennas and can be substituted with $h_{c}^{\prime}(t)$ which is the antenna effects. Practically, $h_{c}^{\prime}(t)$ can be obtained by measuring an empty room without objects. The received signal is therefore given as

$$
r(t)=s(t) *\left\{h_{S}(t) * h_{c}^{\prime}(t)+h_{c}^{\prime}(t)\right\} .
$$

Another choice to filter the antenna effects in the stepped frequency continuous wave (SFCW) radar system is to perform the signal processing technique in the frequency domain. By taking a Fourier transform of (3), the frequency response of the target is derived according to the following equation:

$$
H_{s}(\omega)=\frac{R(\omega)-S(\omega) H_{c}^{\prime}(\omega)}{S(\omega) H_{c}^{\prime}(\omega)},
$$

where $H_{s}(\omega), R(\omega), S(\omega)$, and $H_{c}^{\prime}(\omega)$ are the Fourier transforms of $h_{s}(t), r(t), s(t)$, and $h_{c}^{\prime}(t)$, respectively. So far, one can find the object characteristic by filtering the antenna effects given by (3) or (4).

2.2. Target Identifications. The SEM was introduced by observing the transient response of an object [6]. When an object was illuminated by an ultra-wideband EM wave, the scattering transient response given in (3) can be divided into two successive portions as expressed by

$$
r(t)=r_{\mathrm{ET}}(t)+r_{\mathrm{LT}}(t)+\eta(t),
$$

where $r_{\mathrm{ET}}(t)$ and $r_{\mathrm{LT}}(t)$ denote the early-time and late-time responses of the received signal, respectively. $\eta(t)$ denotes the noise in the system, uncorrelated with either responses. The first response comes from the direct reflection of the incident wave on the object surface. The second one is due to the object's resonance phenomena which can be separated into internal and external modes. The internal resonances are caused by the internal waves experiencing multiple internal reflections while the surface creeping waves cause the external resonance. The signal model of the late-time response from an object can be formulated as a sum of damped exponentials of the following form:

$$
r_{L T}(t)=\sum_{i=1}^{M} R_{i} e^{s_{i} t}+\eta(t),
$$

where $s_{i}=\sigma_{i} \pm j \omega_{i}$ is an $i$ th pole including the damping factor $\sigma_{i}$ and natural frequency $\omega_{i} . R_{i}$ and $M$ are the residues (complex amplitudes) and the number of poles, respectively. The pole is a tool for aspect-independent object identification, depending upon the geometry and physical properties of the object. The commencement of the late-time period coincides with the end of the forced response, due to scattering from discrete object surfaces, and is therefore dependent on the object's geometry and its orientation with reference to the excitation wavefront. On the other hand, the residues are aspect dependent since they represent the coupling between incident electric wave and induced currents on the object. Following the pole extraction using the STMPM in [5], the windowed late-time response can be written as

$$
r_{T}(t)=\operatorname{Re}\left(\sum_{i=1}^{M} R_{i}^{T} e^{s_{i}(t-T)}\right),
$$

in which $T$ is the shifting time and

$$
R_{i}^{T}=R_{i} e^{s_{i} T}=R_{i} e^{\left(-\alpha_{i}+j \omega_{i}\right) T},
$$

Taking the logarithmic scale, (8) can be expressed as

$$
\ln \left(\left|R_{i}^{T}\right|\right)=\ln \left(\left|R_{i}\right|\right)-\alpha_{i} T .
$$

According to (9), the logarithmic magnitude of the residues linearly decreases versus $T$ with slope $\alpha_{i}$. To obtain the damping factor, one can find the slope of the logarithm of the residues in the time frequency scale as well.

\section{Performance Validation via Electromagnetic Simulations}

Simulation examples are illustrated to confirm that the proposed technique, which filters the antenna effects out of the total received backscattering signal, can improve the pole resolution for the radar target identification. The simulations were conducted by using the Higher Order Basic Integral Equation Solver (HOBBIES) EM software. This was done in the frequency domain with the step frequency $(\Delta f)$ of $1 \mathrm{MHz}$. The frequency was swept from $1 \mathrm{MHz}$ to $1200 \mathrm{MHz}$, and the number of the sample frequency $\left(N_{f}\right)$ was 1201 . In simulations, two same antennas were placed together as transmitting and receiving antennas with the spacing of $100 \mathrm{~cm}$. The object was located in the free space at $200 \mathrm{~cm}$ away from the center of the transmitting and receiving antennas. Simulations were divided into four categories regarding to the antenna and object's shape. The antennas which were bow-tie and cone antennas were chosen from the example list of the EM simulation software. The 


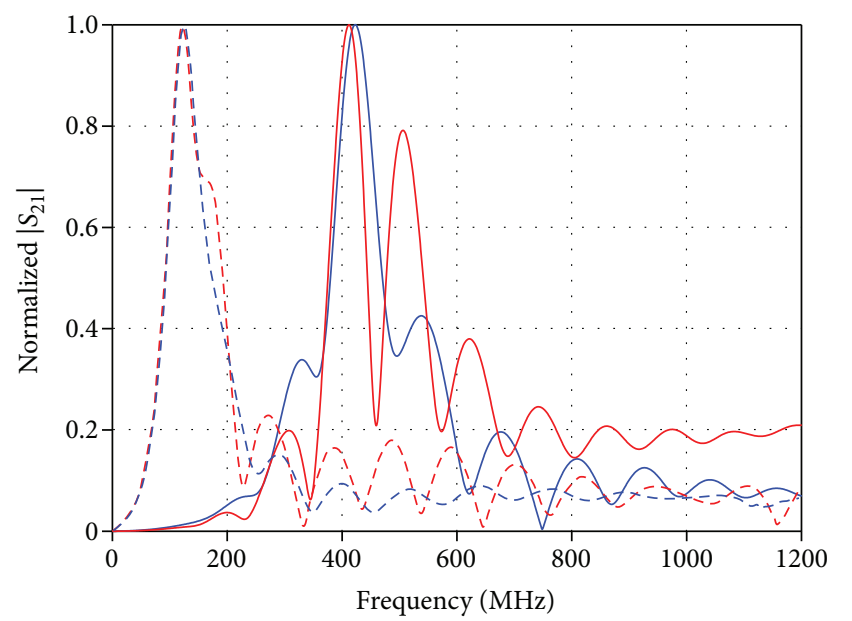

$\begin{array}{lc}\text { - PEC box: bow-tie } & \text { PEC plate: bow-tie } \\ \text { antenna } & \text { antenna } \\ --- \text { PEC box: cone } & --- \text { PEC plate: cone } \\ \text { antenna } & \text { antenna }\end{array}$

FIGURE 2: Frequency response without filtering the antenna effects.

return loss of the bow-tie and cone antennas was below $-10 \mathrm{~dB}$ from $416 \mathrm{MHz}$ to $487 \mathrm{MHz}$ and from $120 \mathrm{MHz}$ to $160 \mathrm{MHz}$, respectively. The bandwidths of these antennas were somewhat narrow, compared with those of the typical radar antenna. In the simulations, the object was constructed with two different shapes, that is, PEC box and plate. The dimensions of the PEC box and plate in $\mathrm{cm}$ were $20 \times 20 \times 30$ and $50 \times 60$, respectively. In this section, simulation examples are distinguished according to the target identification with and without filtering the antenna effects as follows.

3.1. Target Identification without Filtering the Antenna Effects. Figure 2 shows the frequency response obtained from simulations of the target identification without filtering the antenna effects. It is clearly seen that the responses obtained from the use of the bow-tie and cone antennas were significantly different although the same object was under our consideration. On the other hand, the responses of the PEC box and plate were similar when the same antenna type was employed in the simulation. These results were not as expected for target identification. It implies that the antenna was a more important influence than the object's shape.

To confirm the target identification, poles extracted from the scattering responses should be determined. The inverse fast Fourier transform (iFFT) was used to transform the frequency responses to time-domain responses. Poles were extracted from these time-domain responses by using the STMPM. Figures 3(a) and 3(b) show the imaginary (frequency) and real (damping factor) parts of the extracted poles, respectively. In the figure, the frequency shows better stability in the late-time than the damping factor. The damping factor was therefore achieved by finding the slope of the normal logarithm of the residues rather than directly finding the damping factor [5]. Figure 3(c) shows the magnitude of the residues in the logarithm scale. In general, the poles of objects with different shapes and/or constituents should be different. Nevertheless, the figures illustrate that the extracted poles from the PEC box and plate were almost identical for both cases of the bow-tie and cone antennas. The poles of the same object's shape in the simulation with different antennas must be the same; however, the figures showed that the simulation with different antennas achieved different poles. Thus, these poles cannot be directly employed for target identification. This confirms the need to filter the antenna effects leading to the resolution degradation of target identification.

\subsection{Target Identification with Filtering of the Antenna Effects.} In this section, the simulation results are shown to confirm the need to filter the antenna effects. Equation (4) was employed to filter the antenna effects out of the total received response. Poles were extracted again from the time-domain responses by using the STMPM and shown in Figure 4. In the figure, the natural frequency of the PEC box converged to an average of the stable values of $264 \mathrm{MHz}$ and $261 \mathrm{MHz}$ at late-time $t_{\mathrm{LT}}=22 \mathrm{~ns}$ when employing the bow-tie and cone antennas, respectively. The averaged values of the extracted frequency of the PEC plate after the late-time response were $173 \mathrm{MHz}$ and $169 \mathrm{MHz}$, respectively. The damping factor seen in Figure 4(b) begins to stick together when $t_{\mathrm{LT}}=22 \mathrm{~ns}$ with the averaged values of $-6.3 \times 10^{8}$ and $-6.1 \times 10^{8} \mathrm{~Hz}$ for the PEC box and $-3.3 \times 10^{8}$ and $-3.5 \times 10^{8} \mathrm{~Hz}$ for the PEC plate when employing the bow-tie and cone antennas, respectively. Note that the extracted poles including frequencies and damping factors were different when determining the differently shaped objects. Figure 4(c) shows the magnitude of the residues. In the figure, the solid line denotes the range of the constant slope of the line fitted to the natural logarithm of the magnitudes of residues by the linear regression. The slope is constant after $t_{\mathrm{LT}}=22 \mathrm{~ns}$ corresponding to the position of the stability for the extracted natural frequency and damping factor. This is used to confirm the commencement of the late-time response. Reconsidering Figure 4, the shading area denotes the region of the poles extracted from the late-time portions. Poles of the differently shaped objects in different regions showed a significant improvement in the pole resolution.

Table 1 summarizes the averaged pole values obtained from the STMPM with and without filtering the antenna effects. The averaged pole values were calculated from the average of the eighteen samples of the successive poles which were extracted in the late time. In the case of the STMPM without filtering the antenna effects, the averaged poles obtained from the use of the different antennas were significantly different although the same object was under consideration. The averaged poles of different objects were similar when the same antenna type was employed. After using filtering the antenna effects, the averaged poles of the same objects were similar and vice versa although either the same antennas or different antennas were under consideration. Moreover, these poles could be compared with the poles of the objects under plane wave illumination instead of the use of antennas and can be referred to as the reference poles. The table shows that poles obtained from performing the 


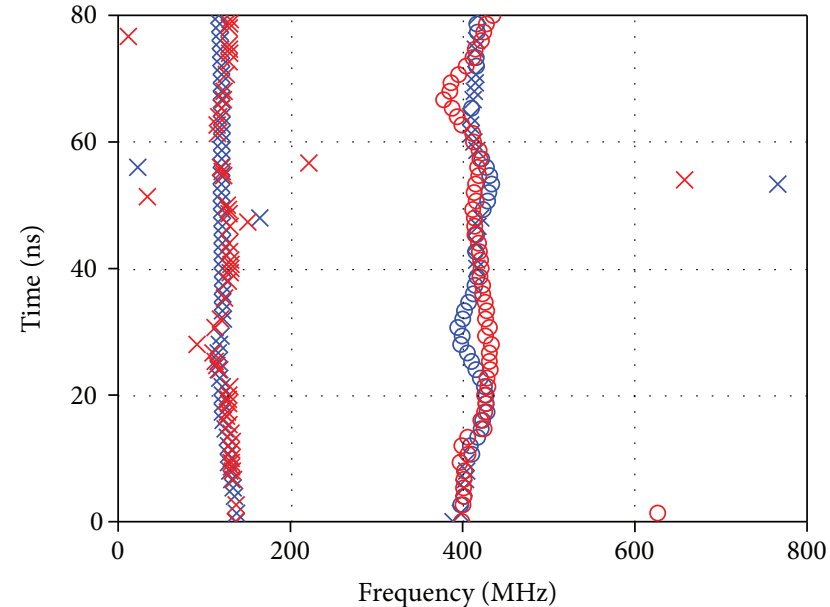

PEC box: bow-tie
antenna
$\times \begin{aligned} & \text { PEC box: cone } \\ & \text { antenna }\end{aligned}$

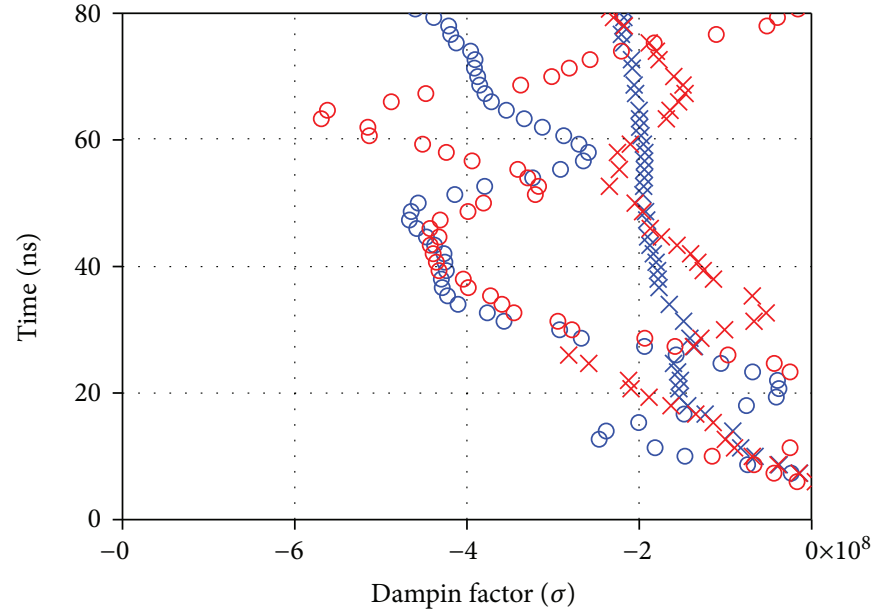

PEC box: bow-tie
antenna
$\times$ PEC box: cone
antenna

PEC plate: bow-tie antenna

$\times \begin{aligned} & \text { PEC plate: cone } \\ & \text { antenna }\end{aligned}$

(a)

(b)

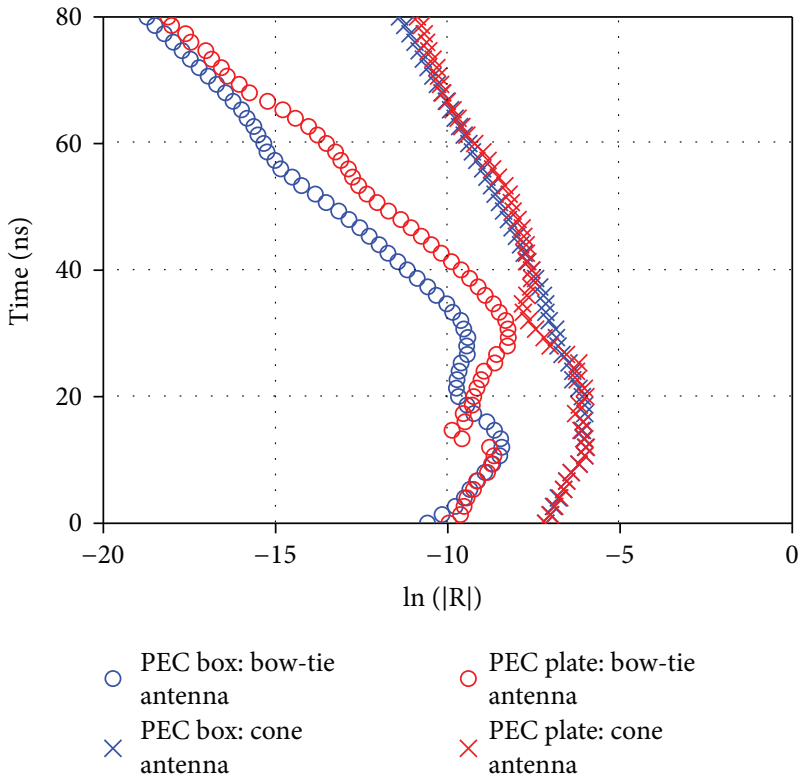

(c)

FIGURE 3: Extracted poles from simulations without filtering the antenna effects; (a) imaginary part (b) real part, and (c) magnitude of residues.

STMPM along with filtering the antenna effects were almost identical to the reference ones. These guarantee that the resolution of the identification was significantly increased when performing the pole extraction along with filtering the antenna effects.

At this end, the resolution of the target identification system must be determined through the Euclidean distance between the poles of the PEC box and plate in the simulations with and without filtering antenna effects. The eighteen samples of the successive poles obtained from the late-time responses of each object were selected for target classification, as shown in Figure 5. The Euclidean distance was calculated from the distance between each averaged pole. The shading symbols denote the averaged value of the poles of each simulation case. The circle line stands for the region of target classification, which was calculated by a half of the Euclidean distance. The Euclidean distances of the pole extraction with and without filtering the antenna were $290 \mathrm{MHz}$ and $51 \mathrm{MHz}$ when employing the bow-tie antennas. The Euclidean distances of $290 \mathrm{MHz}$ and $10 \mathrm{MHz}$ were achieved when employing the cone antennas in the simulations with and without filtering the antenna effects, respectively. The results clearly show that the Euclidean distance for target classification with filtering the antenna effects 


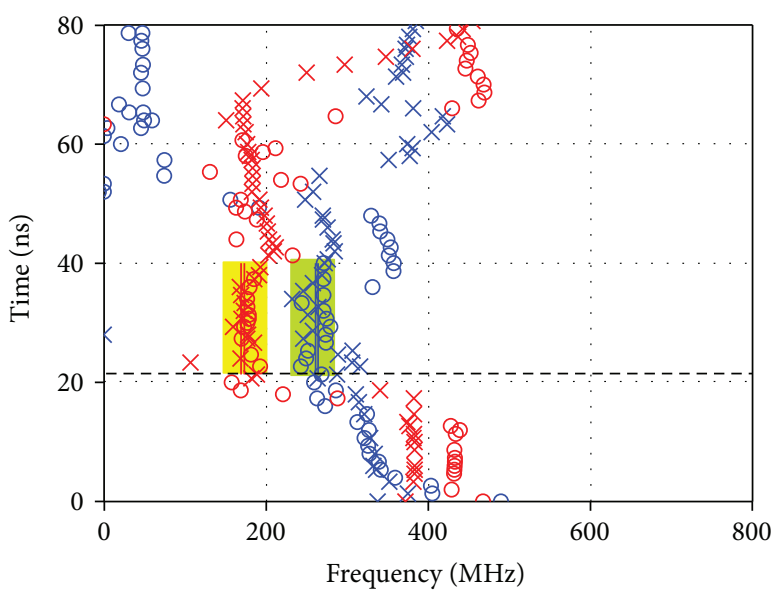

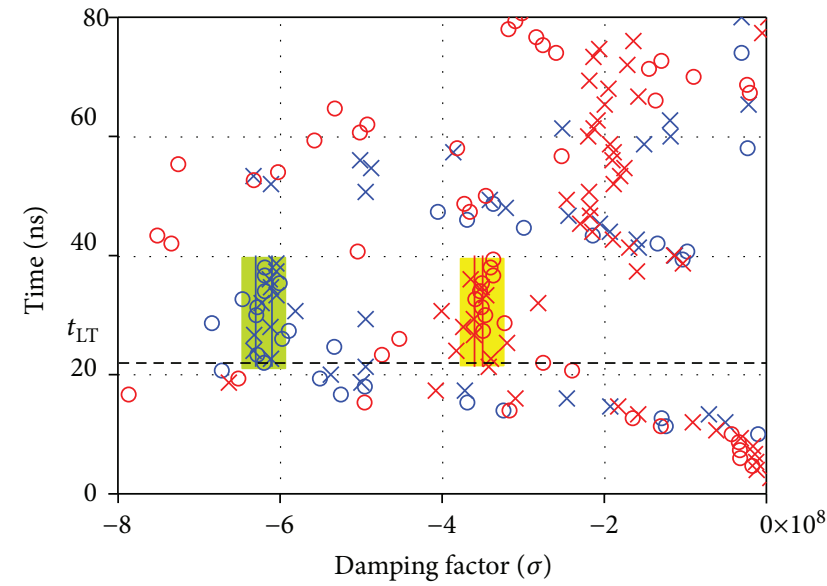

$\begin{array}{ll}\text { PEC box: bow-tie } & \circ \begin{array}{l}\text { PEC plate: bow-tie } \\ \text { antenna }\end{array} \\ \text { antenna } & \times \begin{array}{l}\text { PEC plate: cone } \\ \text { PEC box: cone } \\ \text { antenna }\end{array}\end{array}$

(a)

$\begin{array}{ll}\text { PEC box: bow-tie } & \\ \text { antenna } & \begin{array}{l}\text { PEC plate: bow-tie } \\ \text { antenna }\end{array} \\ \times \begin{array}{l}\text { PEC box: cone } \\ \text { antenna }\end{array} & \times \begin{array}{l}\text { PEC plate: cone } \\ \text { antenna }\end{array}\end{array}$

(b)

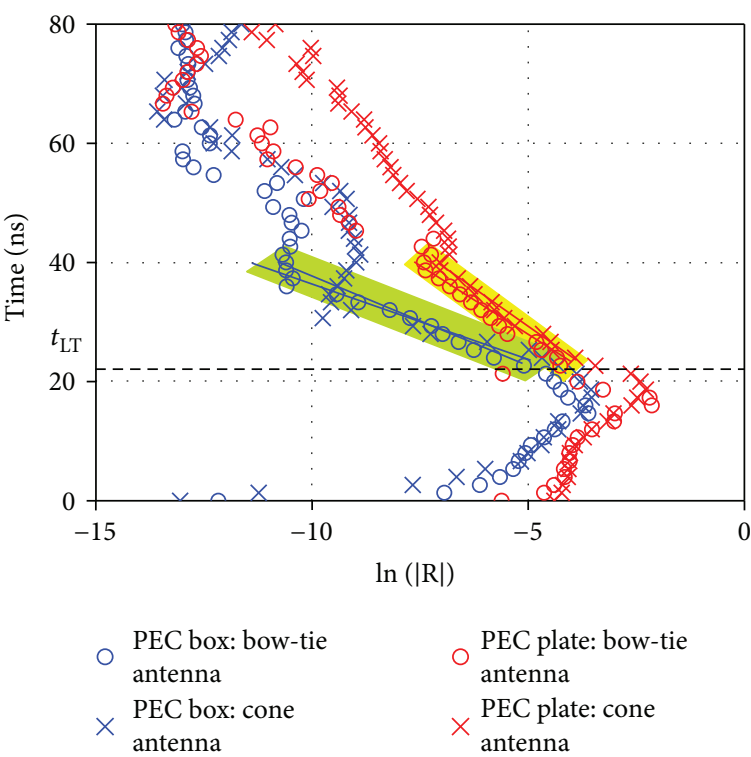

(c)

FIGURE 4: Extracted poles from simulations with filtering the antenna effects; (a) imaginary part (b) real part, and (c) magnitude of residues.

is longer than that of the standard method. This indicates that the resolution of target classification was improved by filtering the antenna effects.

\section{Experimental Validation}

The experimental setup with a bistatic configuration was conducted in order to validate the pole resolution improvement of the radar target identification, as depicted in Figure 6. This was done in an anechoic chamber in order to avoid reflection and other EM waves. For the radar target identification, the results obtained from the simulations and experimentation are not directly compared. Antennas used in the simulations demonstrate the proposed method conceptually. However, in our experiment, log-periodic, bow-tie, and Vivaldi antennas were selected based on the availability at the time of research and their dimensions in $\mathrm{cm}$ were $28.5 \times 23,17 \times 27 \times 13.5$, and $55 \times 70$, respectively. The return loss of the log-periodic [17], bow-tie [18], and Vivaldi [19] antennas was below $-10 \mathrm{~dB}$ in the range of $400-1000,600-4200$, and $240-17,000 \mathrm{MHz}$, respectively. Two of the same antennas were used as transmitting and receiving antennas. The distance between the transmitting and receiving antennas was $60 \mathrm{~cm}$. The object was placed in such a direction that the electric field was propagated at one meter away from the center of the transmitting and receiving antennas. Here, two different shapes of objects, that is, brass plate and box, were determined in order to illustrate the identification. The dimensions of the brass plate and box were $50 \times 60 \mathrm{~cm}^{2}$ and $20 \times 20 \times 30 \mathrm{~cm}^{3}$, 
TABLE 1: Summary of the extracted poles in the late time of simulation results.

\begin{tabular}{lccr}
\hline Pole extraction & Object/antennas & Averaged poles $\left(\times 10^{8}\right)$ & PEC box \\
\hline \multirow{2}{*}{ Without the proposed method } & Bow-tie & $-3.4 \pm j 4.0$ & $-3.6 \pm j 4.3$ \\
& Cone & $-1.3 \pm j 1.2$ & $-1.4 \pm j 1.1$ \\
\hline \multirow{2}{*}{ With the proposed method } & Bow-tie & $-6.3 \pm j 2.6$ & $-3.3 \pm j 1.7$ \\
& Cone & $-6.1 \pm j 2.6$ & $-3.5 \pm j 1.7$ \\
\hline Plane wave illumination & & $-6.8 \pm j 2.6$ & $-3.5 \pm j 1.7$ \\
\hline
\end{tabular}

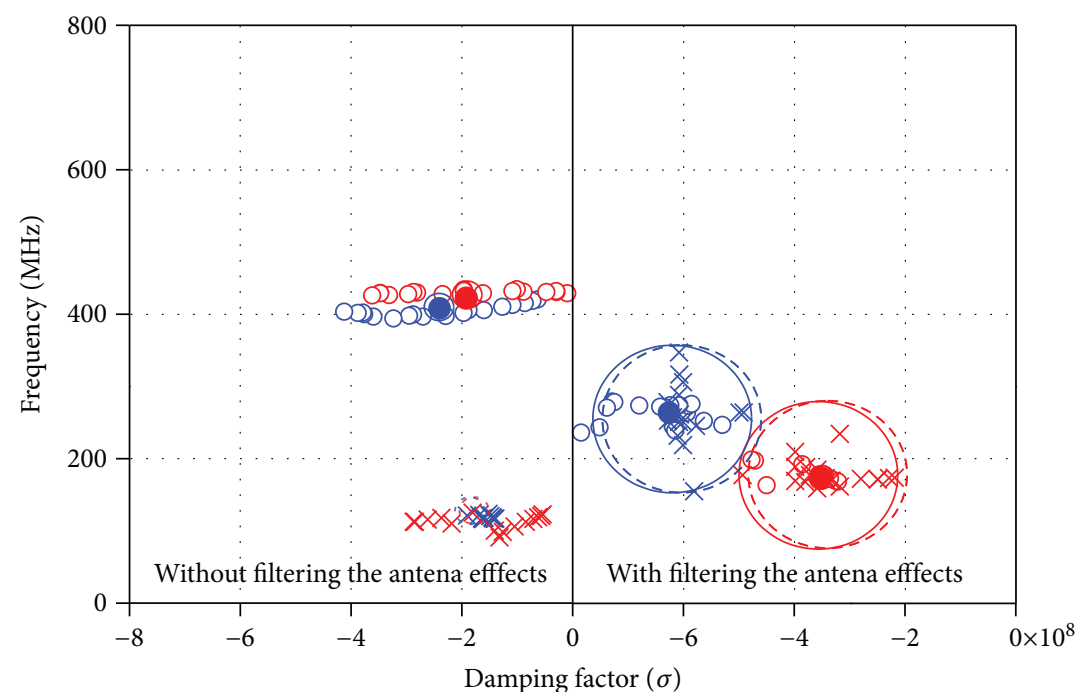
O PEC box: bow-tie antenna
O PEC plate: bow-tie antenna
$\times$ PEC box: cone antenna
$\times$ PEC plate: cone antenna

FIGURE 5: Selected poles of simulations for target classification.

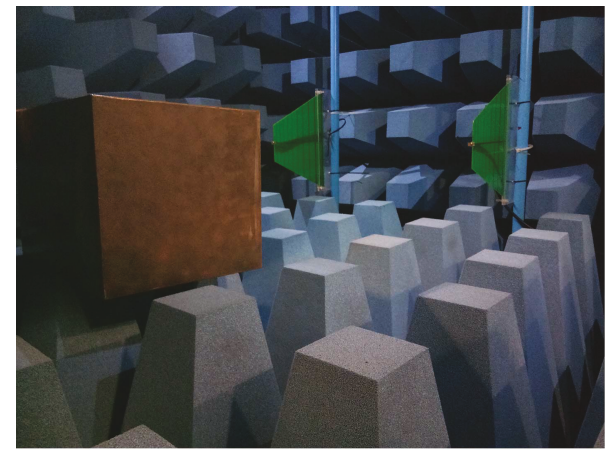

FIGURE 6: Experimental setup of target identification systems.

respectively. A Rohde and Schwarz ZVB20 vector network analyzer was used to perform the experiment. The scattering coefficient $S_{21}$ was measured during the frequency sweep in the range of $200 \mathrm{MHz}$ to $1200 \mathrm{MHz}$ for a total of 101 samples. Each measurement was repeated ten times to achieve accurate results.

4.1. Experimental Results without Filtering the Antenna Effects. Figure 7 shows the measured frequency responses without filtering the antenna effects. The figure clearly reveals

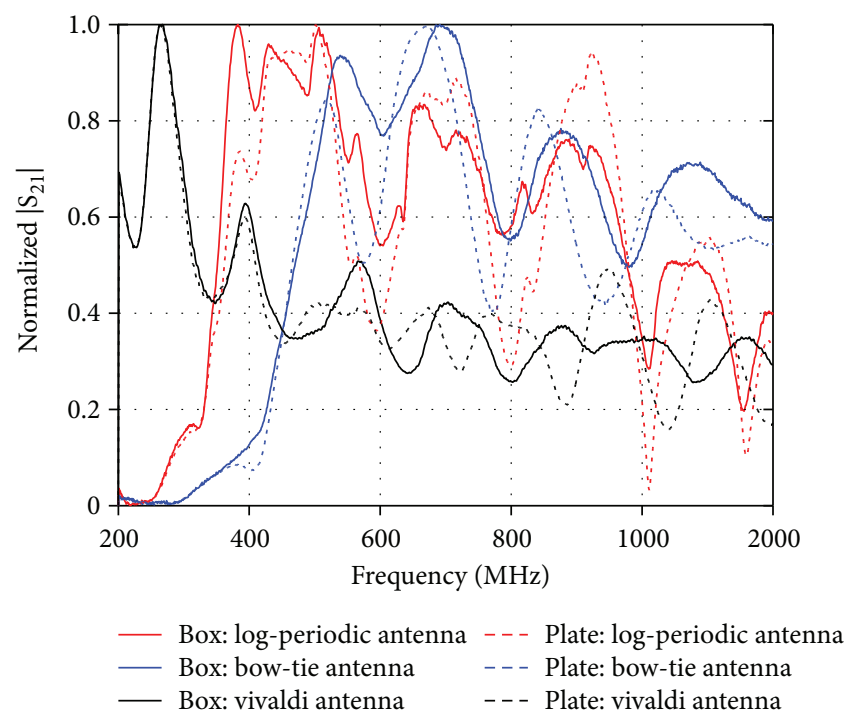

FIGURE 7: Frequency response obtained from experimentation.

that the responses obtained from the use of the same antennas were almost identical although the differently shaped objects were determined. On the other hand, the responses of the same objects were different when employing different 


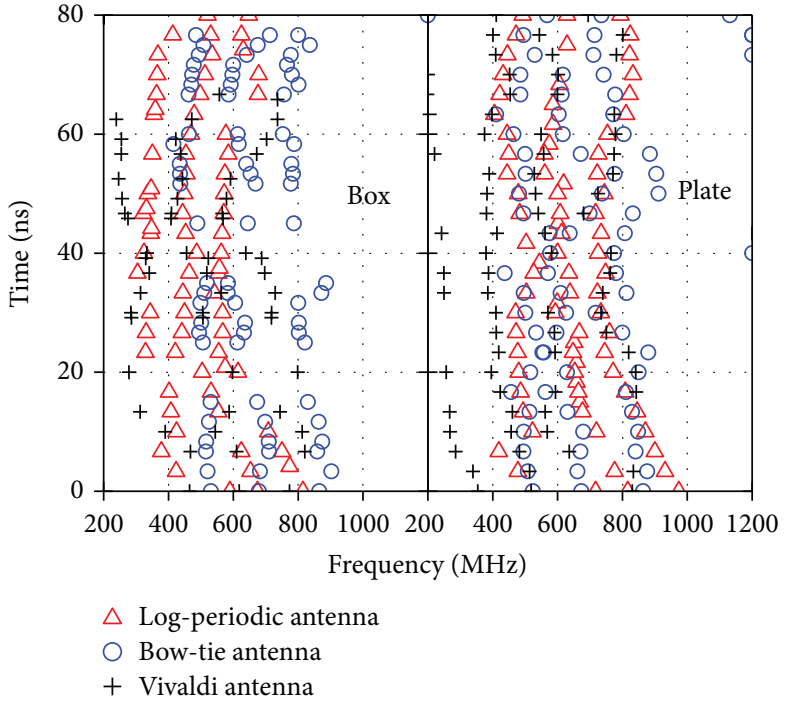

(a)

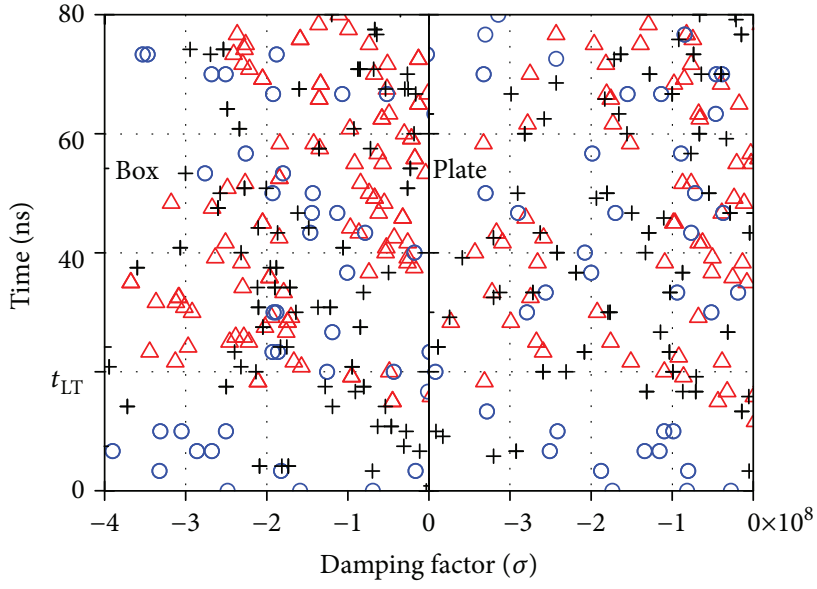

$\triangle$ Log-periodic antenna

$\bigcirc$ Bow-tie antenna

+ Vivaldi antenna

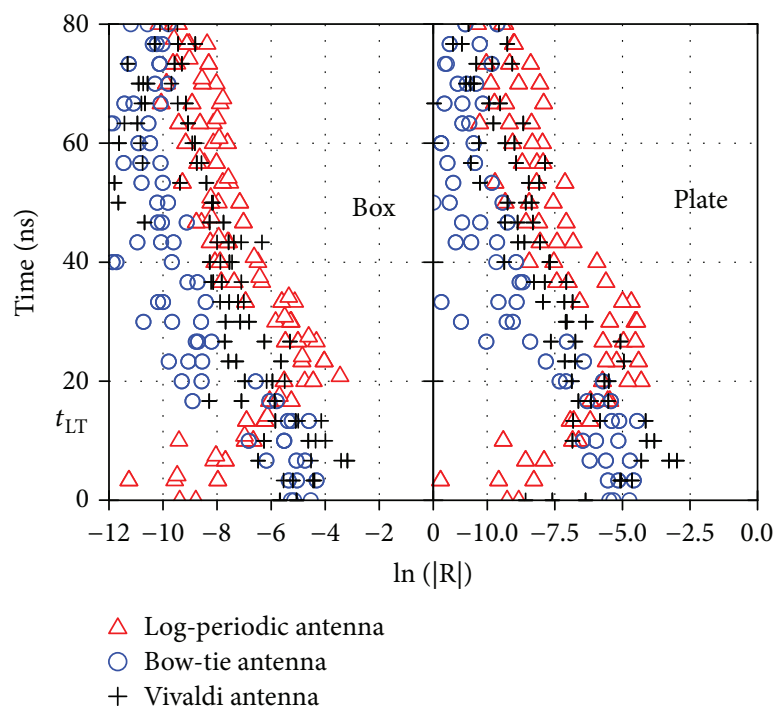

(c)

FIGURE 8: Extracted poles from experimentation without filtering the antenna effects; (a) imaginary part (b) real part, and (c) magnitude of residues.

antennas. The poles extracted by using the STMPM are shown in Figure 8. As seen in the figure, the dispersion in the poles, including the damping factor and frequency of the brass plate and box, was because of the antenna effects and nonidealities in the target identification. The extracted poles should converge to a stable constant value at the commencement of the late-time response [5]. However, in these cases, we could not find the commencement of the latetime responses. Thus, there were no correct poles being employed for the target identification. This guarantees that the problem of the degradation of the identification resolution due to the antenna effects must be resolved.

4.2. Experimental Results with Filtering the Antenna Effects. To improve the pole resolution, the preprocessing technique described by (4) was employed to filter the antenna effects out of the measured frequency response as shown in Figure 7 . The resulting frequency response was then transformed to the time-domain response being used in the process of pole extraction. Figure 9 shows the poles extracted from the experimental results by using the STMPM along with filtering the antenna effects. Compared to the STMPM without filtering the antenna effects, Figure 9 clearly shows that the natural frequencies show a more stable behavior along the time axis. In order to classify the objects, ten samples of the successive poles were selected after the late-time of $t_{\mathrm{LT}}=20 \mathrm{~ns}$. The selected poles were calculated for their averaged values. Table 2 summarizes the averaged pole values. In fact, we could not calculate the averaged pole values in the case of the STMPM without filtering the antenna effects because the certain commencement of the late-time response was nonexistent. In the table, these poles were not applicable 


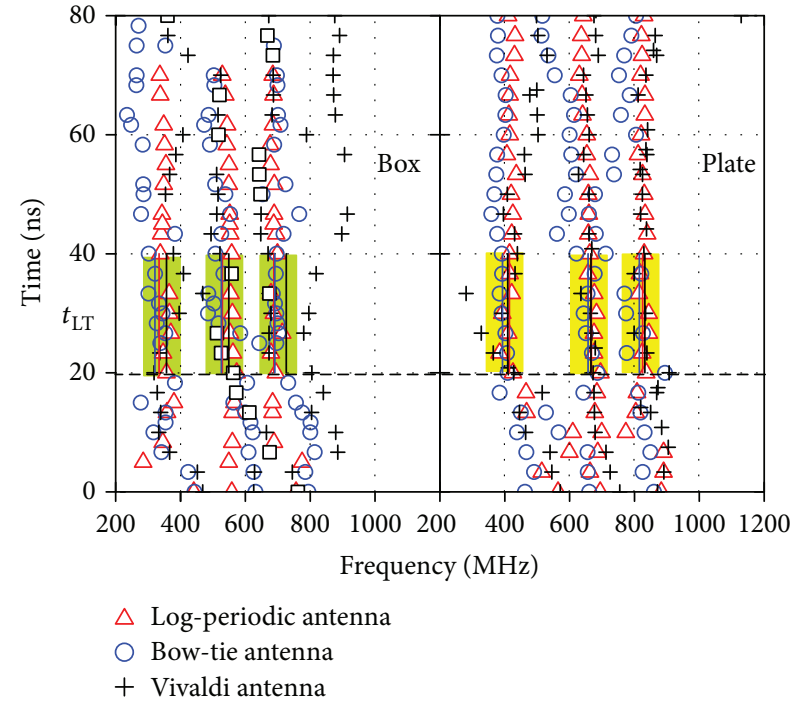

(a)

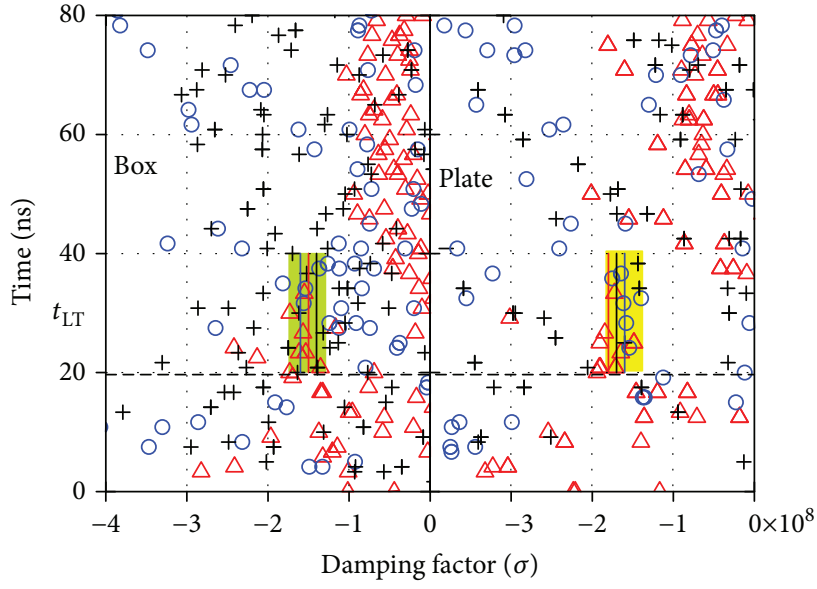

$\triangle$ Log-periodic antenna

O Bow-tie antenna

+ Vivaldi antenna

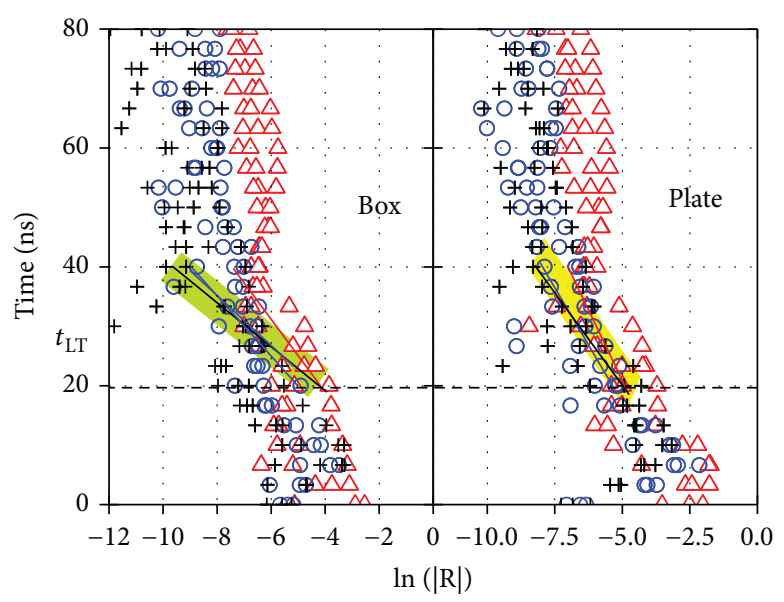

$\triangle$ Log-periodic antenna
$\bigcirc$ Bow-tie antenna
+ Vivaldi antenna

(c)

FIgURE 9: Extracted poles from experimentation with filtering the antenna effects; (a) imaginary part (b) real part, and (c) magnitude of residues.

TABLE 2: Summary of the extracted poles in the late time of experimental results.

\begin{tabular}{lccc}
\hline Pole extraction & Object/antennas & Averaged poles $\left(\times 10^{8}\right)$ & Box \\
\hline & Log-periodic & N/A & N/A \\
Without the proposed method & Bow-tie & N/A & N/A \\
& Vivaldi & $-2.0 \pm j 5.6$ & $-2.0 \pm j 4.1$ \\
& Log-periodic & $-2.1 \pm j 5.6$ & $-2.2 \pm j 4.0$ \\
With the proposed method & Bow-tie & $-2.0 \pm j 5.8$ & $-2.5 \pm j 4.1$ \\
& Vivaldi & N & N \\
\hline
\end{tabular}

(N/A). With filtering the antenna effects, the averaged poles can be achieved, as shown in the table. Note that the poles shown in Tables 1 and 2 were different. The poles obtained from the simulation and experimentation cannot be directly compared. This is because of the identification sensitivity due to the incomplete consistency between the simulation and experimentation conditions such as the antenna type and object material. 


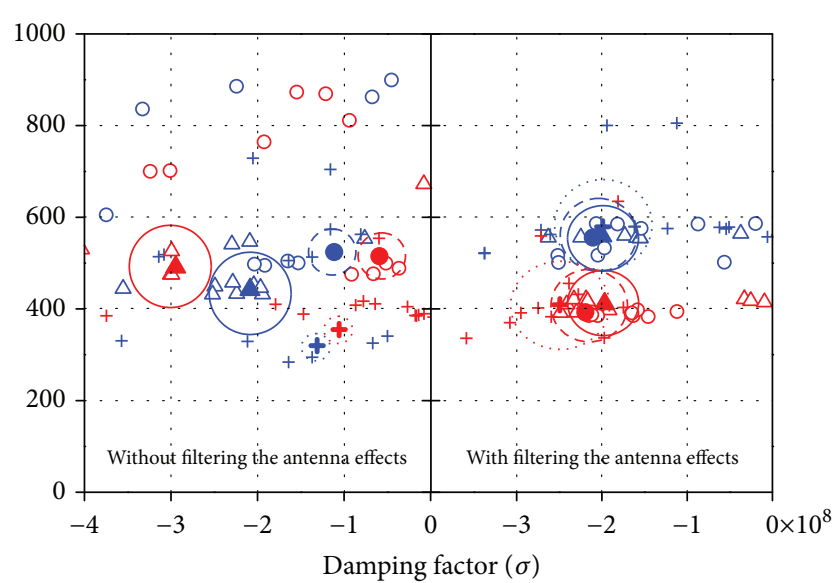

$\begin{array}{ll}\triangle \text { Box: log-periodic antenna } & \triangle \text { Plate: log-periodic antenna } \\ \bigcirc \text { Box: bow-tie antenna } & \bigcirc \text { Plate: bow-tie antenna } \\ + \text { Box: vivaldi antenna } & + \text { Plate: vivaldi antenna }\end{array}$

FIGURE 10: Selected poles of experimentations for target classification.

Figure 10 shows the ten samples of the successive poles extracted after $t_{\mathrm{LT}}=20 \mathrm{~ns}$ by using the STMPM with and without filtering the antenna effects. The shading symbol denotes the averaged pole value of each experimentation case. The Euclidean distances of the averaged poles attained from the STMPM without filtering the antenna effects were $98 \mathrm{MHz}, 53 \mathrm{MHz}$, and $43 \mathrm{MHz}$ when employing the log-periodic, bow-tie, and Vivaldi antennas, respectively. With filtering the antenna effects, the Euclidean distances of $148 \mathrm{MHz}$, $163 \mathrm{MHz}$, and $176 \mathrm{MHz}$ were achieved when employing the log-periodic, bow-tie, and Vivaldi antennas, respectively. The significant difference in the extracted poles of different objects indicates that the STMPM with filtering the antenna effects successfully improved the resolution of the target identification. Furthermore, note that the extracted poles of the use of the commercial off-the-shelf log-periodic antenna whose bandwidth was not ultra-wideband were almost identical to those of the bow-tie and Vivaldi antennas which were designed for the particular purpose when performing the STMPM with filtering the antenna effects.

\section{Conclusion}

In this paper, the investigation of the resolution improvement of radar target identification systems has been presented. The improvement was achieved by filtering the antenna effects due to coupling between the transmitting and receiving antennas and the variation in the antenna response, out of the total backscattering received response. The STMPM was then employed to extract poles from the filtered response. The simulations with different antennas and differently shaped objects were exampled to validate the proposed approach. Furthermore, the experimentations were conducted to confirm the superiority of the proposed approach again. Without filtering the antenna effects, their results showed that one cannot classify objects because extracted poles of differently shaped objects were almost identical. The Euclidean distance of poles of differently shaped objects is larger when performing pole extraction along with filtering the antenna effects. The agreement between simulation and experimental results revealed that filtering the antenna effects can significantly improve the pole resolution.

\section{Conflicts of Interest}

The authors declare that they have no conflicts of interest.

\section{Acknowledgments}

This work has been supported by the Thailand Research Fund through the TRF Senior Research Scholar Program (Grant no. RTA6080008).

\section{References}

[1] M. Khodjet-Kesba, K. Kerroum, C. Faure, K. Drissi, C. Pasquier, and S. Lee, "Comparison of matrix pencil extracted features in time domain and in frequency domain for radar target classification," International Journal of Antennas and Propagation, vol. 2014, Article ID 930581, 9 pages, 2014.

[2] K. Park and K. Kim, "GPR-based landmine detection and identification using multiple features," International Journal of Antennas and Propagation, vol. 2012, Article ID 826404, 7 pages, 2012.

[3] M. Man, Z. Lei, Y. Xie, B. Chen, and Q. Wang, "Monte Carlo simulation of the echo signals from low-flying targets for airborne radar," International Journal of Antennas and Propagation, vol. 2014, Article ID 416985, 7 pages, 2014.

[4] A. Boonpoonga, "Ground penetrating radar (GPR) for counter improvised-explosive devices in Thailand," in 2015 IEEE Conference on Antenna Measurements \& Applications (CAMA), Chiang Mai, Thailand, November-December 2015.

[5] R. Rezaiesarlak and M. Manteghi, "Short-time matrix pencil method for chipless RFID detection applications," IEEE Transactions on Antennas and Propagation, vol. 61, no. 5, pp. 2801-2806, 2013.

[6] C. Baum, On the Singularity Expansion Method for the Solution of Electromagnetic Interaction Problems, 1st Air Force Weapons Laboratory, USA, 1971.

[7] T. K. Sarkar and O. Pereira, "Using the matrix pencil method to estimate the parameters of a sum of complex exponentials," IEEE Antennas and Propagation Magazine, vol. 37, no. 1, pp. 48-55, 1995.

[8] H. Lui and N. Shuley, "Evolutions of partial and global resonances in transient electromagnetic scattering," IEEE Antennas and Wireless Propagation Letters, vol. 7, pp. 436439, 2008.

[9] C. O. Hargrave, I. Vaughan, L. Clarkson, and H. Lui, "Latetime estimation for resonance-based radar target identification," IEEE Transactions on Antennas and Propagation, vol. 62, no. 11, pp. 5865-5871, 2014.

[10] A. Boonpoonga, P. Chomdee, S. Burintramart, and P. Akkaraekthalin, "Simple estimation of late-time response for radar target identification," Radio Science, vol. 52, no. 6, pp. 743-756, 2017. 
[11] N. Chantasen, A. Boonpoonga, S. Burintramart, K. Athikulwongse, and P. Akkaraekthalin, "Automatic detection and classification of buried objects using ground penetrating radar for counter-improvised explosive devices," Radio Science. In press.

[12] C. Abdelhalim and D. Farid, "A compact planar UWB antenna with triple controllable band-notched characteristics," International Journal of Antennas and Propagation, vol. 2014, Article ID 848062, 10 pages, 2014.

[13] T. K. Sarkar, F. I. Tseng, S. M. Rao, S. A. Dianat, and B. Z. Hollmann, "Deconvolution of impulse response from timelimited input and output: theory and experiment," IEEE Transactions on Instrumentation and Measurement, vol. 34, pp. 541-546, 1985.

[14] T. K. Sarkar and F. I. Tseng, "Deconvolution of the impulse response of a conducting sphere by the conjugate gradient method," IEEE Transactions on Antennas and Propagation, vol. 35, pp. 105-110, 1987.

[15] S. Hantscher, A. Reisenzahn, and C. G. Diskus, "Ultra-wideband radar noise reduction for target classification," IET Radar, Sonar \& Navigation, vol. 2, no. 4, pp. 315-322, 2008.

[16] V. A. Mikhnev and P. Vainikainen, "Single-reference nearfield calibration procedure for step-frequency ground penetrating radar," IEEE Transactions on Geoscience and Remote Sensing, vol. 41, no. 1, pp. 75-80, 2003.

[17] "Log periodic printed circuit board antenna 400-1000 MHz," 2017, http://www.wa5vjb.com/products1.html.

[18] A. Sakonkanapong, C. Phongcharoenpanich, and K. Kaemarungsi, "Printed antenna with waning-waxing gibbous shape for pulse transmitter at lower frequency of UWB GPR applications," in 2nd Asia-Pacific Conference on Antennas Propagation (APCAP2013), Chiang Mai, Thailand, December 2013.

[19] R. Natarajan, M. Kanagasabai, and J. George, "Design of an X-band Vivaldi antenna with low radar cross section," IET Microwaves, Antennas \& Propagation, vol. 10, no. 6, pp. 651-655, 2016. 


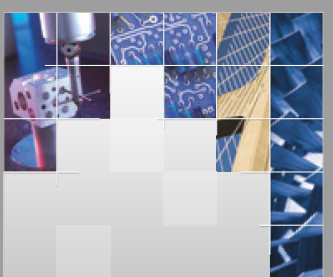

\section{Enfincering}
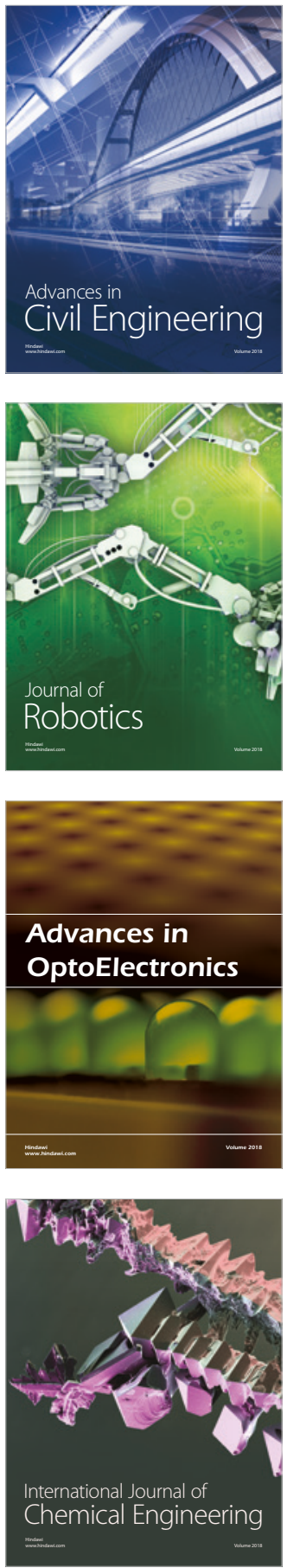

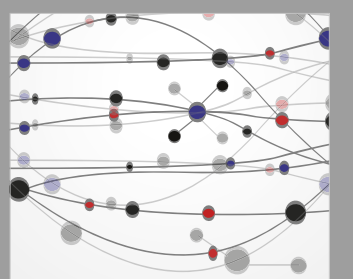

\section{Rotating \\ Machinery}

The Scientific World Journal

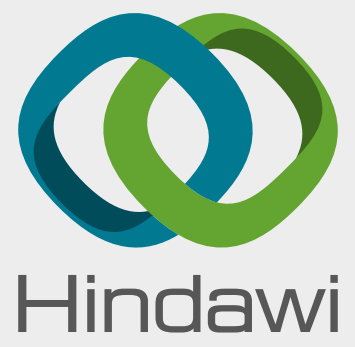

Submit your manuscripts at

www.hindawi.com
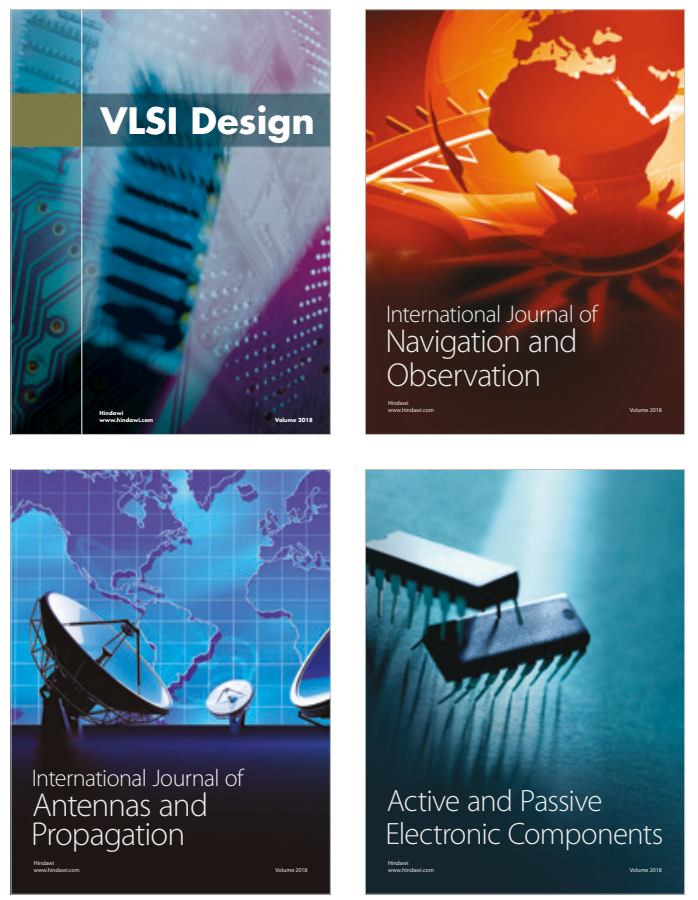
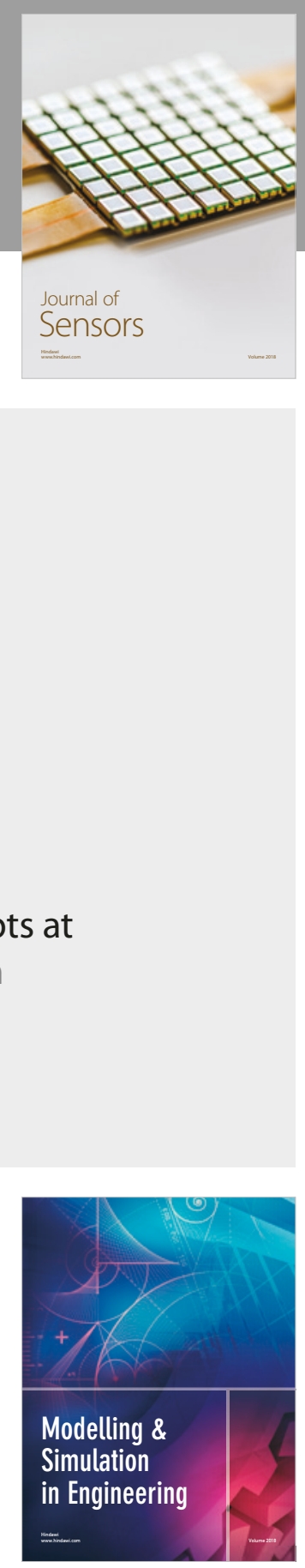

\section{Advances \\ Multimedia}
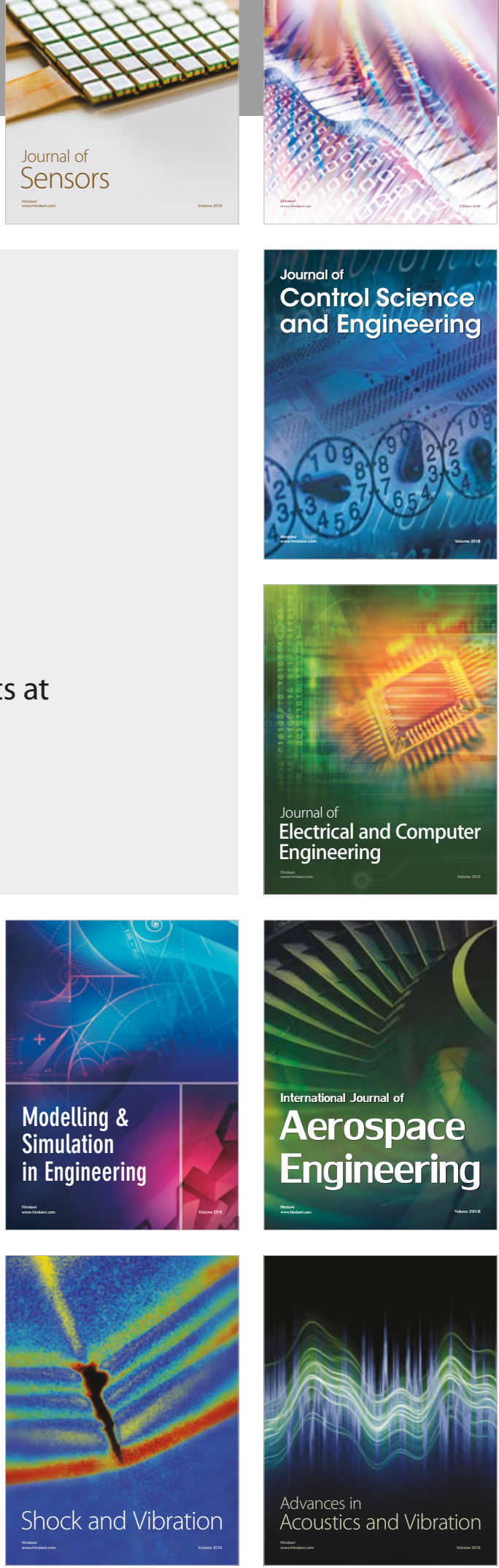